\title{
A CHARACTERIZATION OF MONOMIALS
}

\author{
R. BOJANIC AND W. STOLL
}

A monomial of $n$ complex variables is a function of the form

$$
\begin{array}{ccc}
p_{1} & p_{2} & z_{2} \\
p_{1}^{p_{n}}
\end{array}
$$

where $p_{1}, \cdots, p_{n}$ are non-negative integers and where $a$ is constant. The set

$$
E=\left\{\left(z_{1}, \cdots, z_{n}\right)|| z_{1}|<1, \cdots,| z_{n} \mid<1\right\}
$$

is called the unit polycylinder. The set

$$
D=\left\{\left(z_{1}, \cdots, z_{n}\right)|| z_{1}|=1, \cdots,| z_{n} \mid=1\right\}
$$

is said to be the distinguished boundary of $E$. Note that $D$ is not the whole boundary of $E$.

We want to prove the following theorem:

The monomials are the only entire functions whose absolute value is constant on the distinguished boundary of the unit polycylinder.

Proof. Denote by $\mathbf{C}^{n}$ the space of $n$ complex variables. The elements are the vectors

$$
z=\left(z_{1}, \cdots, z_{n}\right)
$$

whose coordinates $z$, are complex numbers.

Now, let $f$ be an entire function, whose absolute value $|f|$ is constant on $D$. If $|f|$ is identically zero on $D$, then $f$ is identically zero on $\mathbf{C}^{n}$. Therefore, $f$ is a monomial.

We may exclude this case and assume, without loss of generality, that $|f(z)|=1$ for $z \in D$. Now, we want to show that such an entire function $f$ is either constant or has zeros in $\bar{E}$. Assume $f(z) \neq 0$ for $z \in \bar{E}$. Then, we have by a well-known theorem

$$
\begin{aligned}
& \underset{z \in \bar{E}}{\operatorname{Max}}|f(z)|=\operatorname{Max}_{z \in D}|f(z)|=1, \\
& \underset{z \in \bar{B}}{\operatorname{Min}}|f(z)|=\operatorname{Min}_{z \in D}|f(z)|=1 .
\end{aligned}
$$

Therefore, $f$ is constant. Consequently, $f$ is either constant or has zeros in $E$.

Now, we want to prove that $f(z) \neq 0$ for all $z$ in

$$
A=\left\{\left(z_{1}, \cdots, z_{n}\right) \mid z_{1} z_{2} \cdots z_{n} \neq 0\right\} .
$$

Received by the editors February 6, 1961. 
Define the function $g$ by

$$
g\left(z_{1}, \cdots, z_{n}\right)=\overline{f\left(\frac{1}{\bar{z}_{1}}, \cdots, \frac{1}{\bar{z}_{n}}\right)}
$$

on $A$. This function is holomorphic on $A$ since its real partial derivatives exist, are continuous, and satisfy the Cauchy-Riemann equations

$$
g_{z_{\nu}}\left(z_{1}, \cdots, z_{n}\right)=\overline{f_{z_{\nu}}\left(\frac{1}{\bar{z}_{1}}, \cdots, \frac{1}{\bar{z}_{n}}\right) \frac{\partial}{\partial z_{\nu}}\left(\frac{1}{\bar{z}_{\nu}}\right)}=0 .
$$

For $z \in D \subset A$, we have

$$
\begin{aligned}
g\left(z_{1}, \cdots, z_{n}\right) & =\overline{f\left(\frac{1}{\bar{z}_{1}}, \cdots, \frac{1}{\bar{z}_{n}}\right)}=\overline{f\left(z_{1}, \cdots, z_{n}\right)} \\
& =\frac{1}{f\left(z_{1}, \cdots, z_{n}\right)} .
\end{aligned}
$$

Therefore

$$
g\left(z_{1}, \cdots, z_{n}\right) \cdot f\left(z_{1}, \cdots z_{n}\right)=1 \quad \text { for }\left(z_{1}, \cdots, z_{n}\right) \in D .
$$

The function $g \cdot f$ is holomorphic on $A$ and identically one on $D$. Since $A$ is a connected, open neighborhood of $D$, the function $g \cdot f$ is equal to one on $A$ :

$$
f(z) \cdot g(z)=1 \quad \text { for } z \in A .
$$

Therefore, we have $f(z) \neq 0$ for $z \in A$.

Our function $f$ vanishes at most on the planes $\left\{\left(z_{1}, \cdots, z_{n}\right) \mid z_{p}=0\right\}$. Therefore $f$ has the form ${ }^{1}$

$$
f\left(z_{1}, \cdots, z_{n}\right)=z_{1}^{p_{1}} \cdot z_{2}^{p_{2}} \cdots z_{n}^{p_{n}} \cdot h\left(z_{1}, \cdots, z_{n}\right)
$$

where $p_{1}, \cdots, p_{n}$ are non-negative integers and $h$ is an entire function which does not vanish at all, and whose absolute value is constant on $D$. Consequently, $h$ is a constant. We obtain

$$
f\left(z_{1}, \cdots, z_{n}\right)=a z_{1}^{p_{1}} \cdots z_{n}^{p_{n}}
$$

q.e.d.

\section{University of Notre Dame}

'See: Osgood, Lehrbuch der Funktionentheorie, Vol. III, Chapter III, \$26. 